\title{
5G NR over satellite links: Evaluation of synchronization and random access processes
}

\author{
Harri Saarnisaari ${ }^{1}$, Senior Member, IEEE, Carlos Morais de Lima, Member, IEEE \\ University of Oulu, Oulu, Finland \\ Tel: (+358) 405727803,e-mail: harri.saarnisaari@oulu.fi
}

\begin{abstract}
Integration of $5 \mathrm{G}$ new radio (NR) access interface with satellite technology is an ongoing task in the standardization bodies. Since $5 \mathrm{G}$ NR was originally designed as a terrestrial system, its direct suitability to satellite communications has yet to be carefully evaluated. In that regard, the initial synchronization and random access processes are among the topics of particular interest. This paper overviews these key processes by first identifying problematic points and then proposing feasible solutions. It is shown that initial synchronization and related data demodulation of the $5 \mathrm{G}$ NR synchronization block is possible using dedicated large frequency shift aware receivers. In addition, it is shown that timing advance value, which is a part of the random access process, has to be extended to cover satellite delays. A few ways to do this are discussed in the paper, as well as ways to reduce capacity loss due to required large guard interval of the $5 \mathrm{G} \mathrm{NR}$ random access signal.
\end{abstract}

Keywords: initial access, random access, mobile networks, satellite systems.

\section{INTRODUCTION}

Both terrestrial mobile networks and satellite systems would benefit if their cooperation could be tightened. So far, these different systems have been designed separately and co-operation has been based on gateway solutions such that, for example, satellite systems are able to support 4G systems. Since early 2018 the satellite element has been included into the 3GPP 5G new radio (NR) standardization work [1]. This should result in more fluent usage of satellites complying with $5 \mathrm{G}$ systems specifications. The identified use cases are backhaul usage and direct user access. Integration should be done in all levels. Currently, key technologies introduced by the 5G NR are still undergoing integration, for instance, high level softwarization of network functions, related management and orchestration as well as distributed network functions, slicing, multi access edge computing among others.

Another level of integration is the use of 5G NR in satellite systems. This raises new problems since 5G NR is designed for terrestrial operations. While the satellite link budget is not a concern, the long propagation delays and large Doppler frequency shift values are the main difference between space and terrestrial domains. In fact, the delay is the main feature affecting not only all the aforementioned functionalities, but also the medium access (MAC) and physical layers. However, the Doppler frequency shift mainly affects the physical layer that has to compensate it before signal reception could be successful.

In this paper we provide a summary of the user equipment' (UEs') initial access under severe Doppler frequency shift. Indeed, this is about reception and demodulation of the base station's (gNB's) synchronization signal (SS) block [2]. In addition, we provide a summary of the random access process under long satellite delays. In the both cases we briefly describe the process in 5G NR, the problems and possible solutions. These overviews are based on the work done in the SaT5G project [http://sat5g-project.eu/] and will be published with more details in following papers and upcoming deliverables.

In $3 \mathrm{GPP}$, satellite connections are under non-terrestrial networks (NTN) [1] specifications, and satellite capable receivers are called NTN terminals. They could be either UEs or satellite receivers with gNBs in the backhaul case. This paper covers both use cases, namely initial and random access, and refers to receivers as NTN terminals. The respective transmitter is either a satellite or a satellite ground element. Notice that the former is valid for satellite systems with on board processing capability such that gNB functions (or some of them) are in the satellite, While the latter is valid for pent-pipe satellites where the satellite operates as an amplify-andforward relay platform.

\section{SATELLITE SYSTEM CHARACTERISTICS}

In this section we shortly describe the satellite system specialities mentioned in the introduction. First, there are two kinds of satellite systems. Pent-pipe satellites are amplify-and-forward systems whereas regenerative or onboard processing satellites process the signal and may included base station properties. The satellites are stable at geostationary orbit (GEO) and mobile at medium, low and highly elliptical orbits (MEO, LEO and HEO).

The main differentiators are large delay and Doppler frequency shift. LEO satellites are at $600 \mathrm{~km}$ and up whereas GEO satellites are at about $36000 \mathrm{~km}$. At $3 \mathrm{GHz}$ and $30 \mathrm{GHz}$ carriers, the satellite Doppler frequency

\footnotetext{
${ }^{1}$ The work has been financially supported by EU Horizon 2020 project SaT5G under grant agreement No
} 761413, and Academy of Finland 6Genesis Flagship (grant 318927). 
shift could be $\pm 50 \mathrm{kHz}$ and $\pm 720 \mathrm{kHz}$, respectively, and corresponding frequency change rates $600 \mathrm{~Hz} / \mathrm{s}$ and 8.2 $\mathrm{kHz} / \mathrm{s}[1]$.

\section{INITIAL ACCESS}

Initial access means that a UE tries to detect existing base stations and read information about access parameters. These are provided in the SS block send by the base stations that contains the primary and secondary synchronization signals (PSS and SSS) as well as the system information data that is heavily coded using a polar code [2].

One of the driving forces in mobile technology development has been simplicity of the user device. This means that tolerated carrier frequency uncertainty or offset (CFO) has been limited such that a simple initial synchronization algorithm could be applied. However, these cannot stand large CFO since they fail to detect the signal in that case. Furthermore, it is well known that orthogonal frequency division multiplexing (OFDM) signal are sensitive to CFO that are more than a few percent of the SCS since the bit error rate (BER) or block error rate (BLER) increase with increasing CFO.

The detection is about detecting the PSS whose duration $T$ varies depending on the SCS. Let $F$ denote the CFO. It is known that the detection sensitivity starts to drop once $F T>0.1$ and signal-to-noise ratio (SNR) loss is $2 \mathrm{~dB}$ if $F T=0.35$ [3]. In OFDM systems, $F T=1$ if CFO is equal to the SCS such that sensitivity losses start to occur once CFO is $20-30 \%$ of the SCS. In satellite systems, this may be the case depending on satellite velocity.

This is well known problem in global navigation system (GNSS) receivers which is solved by employing multiple receivers instead of just one. Each receiver is tuned to different CFO. The frequency separation between the receivers is based on expected losses as explained above. Tighter separation is needed if only minor losses can be tolerated. The receiver structure is shown in Figure 1. This is against the "simple handheld" design principle such that more powerful receivers are needed in satellite systems where larger Doppler frequency shift is a concern.

In addition to PSS detection, also the SSS must be detected, and data decoded. After initial CFO estimation in the PSS detector, frequency uncertainty is limited down to an acceptable level and the SSS can be detected. The data part needs fine tuning the CFO estimate but standard methods, like the use of cyclic prefix or training symbols based CFO estimators, are usable herein.

With the described receiver, it is possible to detect the PSS and SSS as well as decode the data when SNR per resource element (RE) (or active subcarrier) is negative. Indeed, reliable PSS (and SSS) detection is possible down to $-6 \mathrm{~dB}$ SNR/RE that is $5 \mathrm{G}$ NR requirement for this. Depending on SNR, reliable data detection may require a few receptions of the SS block depending on CFO estimation and channel estimation accuracy.

More information about these SS block reception aspects could be find in [4] that includes also simulation results that confirm the above statements as well as contain additional references.

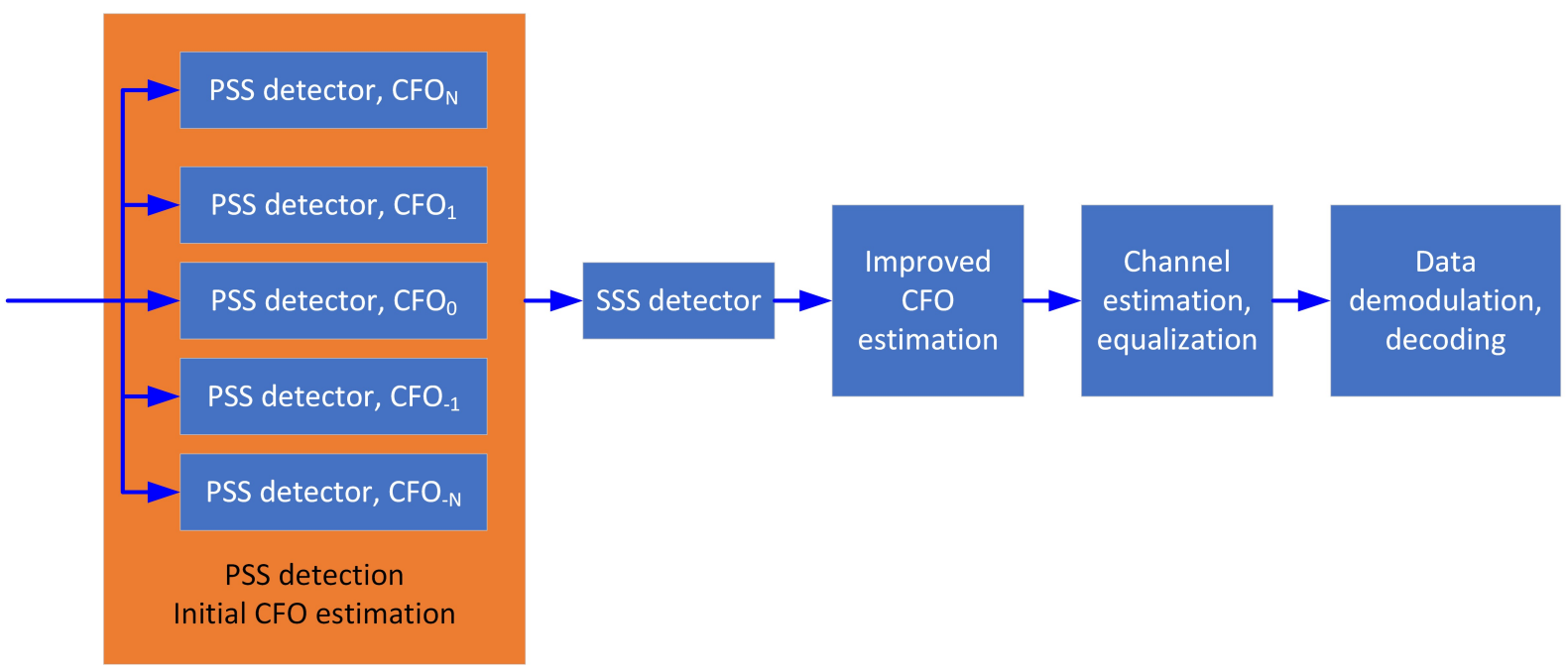

Figure 1. A receiver structure for the SS block processing with severe Doppler frequency shift.

\section{RANDOM ACCESS}

The random access (RA) procedure is used to: $i$ ) establish synchronization to new cells when performing handover; $i$ i) resynchronize to the current service cell due to long periods of UL inactivity; and iii) to request UL scheduling if dedicated scheduling-request resource has not been established yet. A non-terrestrial network (NTN) terminal still in the IDLE mode employs the RA process to connect to a new cell during the initial access, 
or to request resources for uplink transmission, as well as to re-establish uplink synchronization in the CONNECTED mode. The RA procedure is carried out in either contention based or contention free manner: while the former is employed when a UE is not yet synchronized or lost its synchronization, the latter is applied when a UE has already synchronized with a serving gNB. Both procedures rely on preamble transmissions from UEs to the serving gNB [5]. The preamble is transmitted on specific time/frequency resources which are indicated by gNB and sent to UEs over control channels. The 5G NR RA procedure is illustrated in Figure 2.

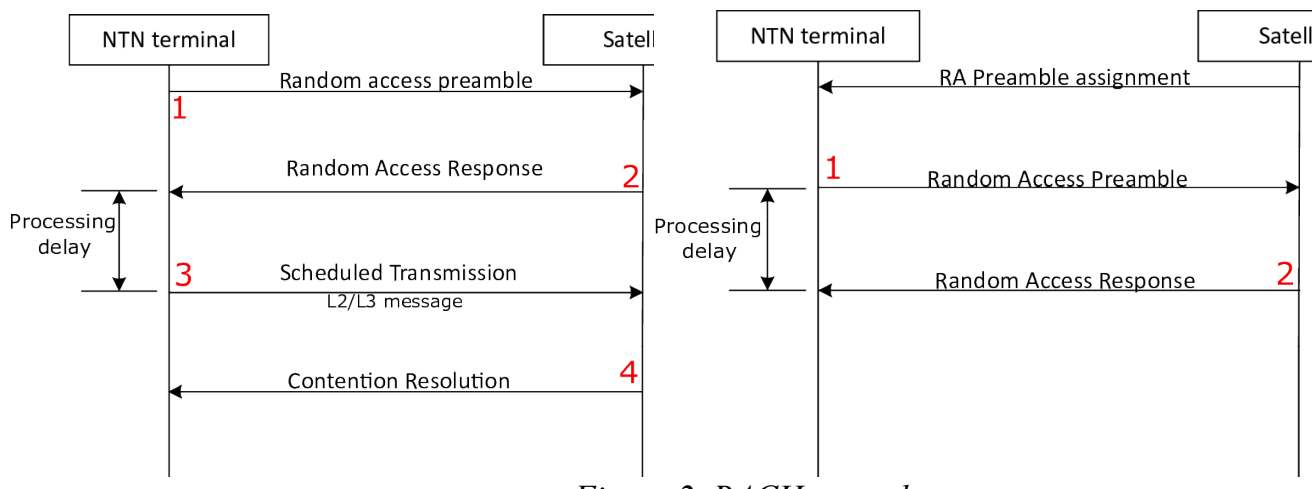

Figure 2. RACH procedure.

It is worth noticing that fast moving satellites could also include gNB functions as opposed to terrestrial systems where base stations are static. As a result, handovers in satellite systems may be triggered by both gNB and UE mobility. Hereafter, we identify which technical challenges may hinder the usage of the terrestrial $5 \mathrm{G}$ NR RACH process in the satellite systems and then propose viable solutions which comply with current standards.

\subsection{PRACH Guard Interval}

The physical random access channel (PRACH) carries the UE RA preamble (signal 1 in Figure 2) for the UL synchronization. In fact, the UE sends its RA preamble within a RACH slot using the previously received SS block as the timing reference. Therefore, the preamble reception timing varies unpredictably as function of the propagation delay within the cell. In $5 \mathrm{G} \mathrm{NR}$, the gNB scheduler needs to ensure that the preamble reception does not overlap other transmissions [2]. Thus, the scheduler incorporates extra guard interval (GI) to capture such uncertainties. It is worth mentioning that unlike previous generations, $5 \mathrm{G}$ NR does not specify the guard interval; therefore, it can be adapted to match different cell sizes, even those in satellite communications.

The GI value depends on the round-trip time (RTT) between the satellite and the longest link to the beam coverage area (or footprint). The RTT experienced in satellite systems is much larger than in terrestrial systems varying from $4 \mathrm{~ms}$ for low Earth orbit (LEO) satellites at $600 \mathrm{~km}$ altitude to hundreds of ms for geostationary orbit (GEO) satellites. This means that the scheduler has to reserve significant amount of resources just for GI, which is the principal problem concerning this subsection.

One way to reduce the required GI is to use so called differential distance (DD) concept. Herein, the shortest distance from the satellite to the footprint is transmitted to the NTN terminal and they can subtract it. The remaining delay that has to be covered by GI is the difference between the maximum one and this minimum. The concept is illustrated in Figure 3. The minimum distance could be per beam and it could be sent dynamically since the beam shape may change. The minimum distance would be a new system information that needs to be defined in the $5 \mathrm{G}$ NR standard. The maximum footprints are from a few hundred $\mathrm{km}$ up to several thousand $\mathrm{km}$ [1].

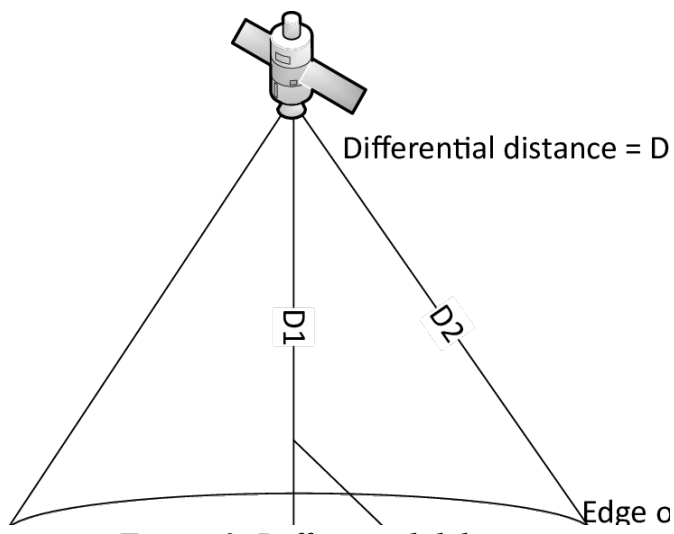

Figure 3. Differential delay. 
It is also be possible to reduce capacity the PRACH needs by thinning its intervals, especially with the DD concept. Currently, RACH slots periodicity may vary from $10 \mathrm{~ms}$ to $160 \mathrm{~ms}$ (Section 16.2)[2]. This is a viable option since handovers do not necessarily occur very frequently in satellite systems, especially in backhaul configuration where NTN terminals (as part of gNB) tend to have almost continuous backhaul needs.

\subsection{Timing Advance Value}

In response to the RA preamble, the serving gNB sends a random access response (RAR) message (signal 2 in Figure 2) consisting timing advance (TA) value among other things. The UE is awaiting this message a time determined by a RAR response timer. This timer and other related ones have to be enlarged to cope the satellite delays.

The TA value is calculated according to guidelines in (Section 4.2)[5]. The largest TA corresponds $300 \mathrm{~km}$ cell size RTT at $15 \mathrm{kHz}$ sub carrier spacing (SCS). At $30 \mathrm{kHz}$ SCS this is $150 \mathrm{~km}$ and so on. The RAR message has 12 bits reserved for TA value. Obviously, these values are often too small for satellite systems since they will probably use larger SCS values. In what follows, we propose two methods to extend the TA range and cope with the aforesaid limitations.

The first method is straightforward and implemented by direct extension of the maximum TA range. This range depends on a used satellite system (altitude, footprint) as well as used SCS. The required extra bits should be included into the RAR message or other mean to transmit them is required.

The second method applies the DD concept but now we call it a differential delay concept. If the satellite footprint is small, extra bits for TA value are not needed but often the footprints are larger and extra bits are needed. The benefits of this alternative, in terms of extra bits, are most obvious for higher altitude satellite systems like GEO and medium Earth orbit (MEO) satellite systems since in those the delay reductions are larger. However, since the DD concept is beneficial also in reducing the required GI, this may be an interesting option.

More detailed analysis of these random access issues can be found in [6].

\section{CONCLUSIONS}

This paper overviews the 5G NR initial synchronization and random access processes applied to satellite links. In particular, challenges and viable solutions to the observed problems were discussed. Large Doppler frequency shift is undergone in some satellite systems meaning that in those cases the standard initial synchronization receivers cannot be used. As a result, dedicated large (and more complicated) CFO aware receivers are needed. Large delays experienced by satellite systems cause problems for the random access process. The timing advance value has to be extended is most cases and process related timer values enlarged accordingly. However, the guard interval process in 5G NR is flexible and can be adapted to the longer satellite delays at expense of overall system capacity as previously addressed.

\section{ACKNOWLEDGEMENTS}

This paper is based on results obtained in the Horizon 2020 SaT5G project under grant agreement No 761413 that aims to study integration of satellite and $5 \mathrm{G}$ terrestrial systems in a wide sense, from many angles. This research has also been supported by Academy of Finland 6Genesis Flagship (grant 318927).

\section{REFERENCES}

[1] 3rd Generation Partnership Project; Technical Specification Group Radio Access Network, Study on new radio (NR) to support non terrestrial networks (release 15), 2018, 3GPP TR 38.811 V15.0.0 (2018-06).

[2] E. Dahlman, S. Parkval, and J. Sköld, 5G NR: The Next Generation Wireless Access Technology, Academic Press, 2018.

[3] O. C. Maus, F. Classen, and H. Meyr, Carrier frequency recovery for a fully digital direct-sequence spreadspectrum receiver: A comparison, in Proceedings of the IEEE Vehicular Technology Conference, 1993, pp. 392-395.

[4] H. Saarnisaari, J-M. Houssin, T. Deleu: 5G New Radio Over Satellite Links: Synchronization Block Processing, submitted to EuCNC, 2019.

[5] 3GPP TR 38.300 V15.0.0, "Technical Specification Group Radio Access Network; NR; NR and NR-RAN Overall Description; Stage 2 (Release 15)," Technical Report, 2018.

3GPP TR 38.213 V15.0.0, "Technical Specification Group Radio Access Network; NR; Physical layer procedures for control (Release 15)," Technical Report, 2018.

[6] H. Saarnisaari, A. Laiyemo, C. M. de Lima: Random Access Process Analysis of 5G New Radio Based Satellite Links, to be submitted to Satellite and Non-Terrestrial Networks for $5 G$ Workshop in IEEE $5 G$ World Forum, 2019. 